\title{
Cloning antibodies from serum
}

Proteomics analysis of polyclonal antibodies guides monoclonal production.

When faced with a foreign invader, mammals launch a diverse defense. One component is an array of antibodies, each binding the antigen in its own way, each generated from a clonal population of white blood cells called B cells. New work shows how to identify and manufacture the best of these antibodies in a reproducible way.

Researchers can generate antibodies against antigens by inoculating an appropriate animal (usually a rabbit) and then collecting the desired antibodies from its blood. 'Polyclonal antibodies' comprise the collection of all antibodies against a given antigen produced in this fashion. Unfortunately, polyclonal antibodies are not ideal for many research and medical applications because their properties are hard to define and reproduce consistently. For this reason, antibodies that are produced by just one clone of $\mathrm{B}$ cells, or 'monoclonal antibodies', are often preferred. However, generating effective monoclonal antibodies can be difficult and time consuming, and when using traditional means for this, such as hybridomas, much of the antibody repertoire is lost.

Researchers at Cell Signaling Technology in Danvers, Massachusetts have now used proteomics to identify the antigen-binding sequences of promising antibodies produced by inoculated animals and then clone promising sequences to generate monoclonal antibodies.

Purifying high-affinity antibodies produced in an inoculated mouse or rabbit is straightforward-these bind most tightly to an antigen and can readily be captured. However, analyzing the sequences of these proteins is more challenging. A typical proteomics analysis involves enzymatically digesting collected proteins into peptide fragments and then characterizing them by mass spectrometry. Reference databases based on gene collections can predict which fragments are likely to be produced by a particular protein digested by a particular enzyme and so identify proteins in the sample. Because immune systems shuffle portions of the genes to produce a dizzying assortment of antibodies, however, these genes are not accurately represented in the reference databases. To get around this problem, the researchers created a specialized reference database using next-generation sequencing. After inoculating rabbits with a human peptide associated with breast cancer, the researchers selected the animal with the best response, collected RNA from its white blood cells and sequenced transcripts coding for antibody chains.

The researchers also collected the highestaffinity antibodies from the rabbit's serum. These were divided into aliquots, each digested with different enzymes, producing unique sets of digestion fragments that could be analyzed by mass spectrometry and compared to each other.

To deduce the sequences in the bestbinding antibodies reliably, the researchers considered only data with particularly high coverage of the antibody regions and at least a dozen unique peptides. This analysis could identify likely sequences for antibody regions known as the heavy and light chains but not which sequences occurred together in the same antibody. Thus, the researchers expressed and tested multiple possibilities. This resulted in two monoclonal antibodies, both with better specificity and sensitivity than the original polyclonal antibody mixture.

Researchers have used this technique of inoculating animals and creating antigenspecific reference libraries to develop antibodies from inoculations in both rabbits and mice. The technique has now been used to create antibodies from over a dozen antigens, says company CSO Roberto Polakiewicz.

Traditional methods of developing monoclonal antibodies require constructing and interrogating thousands of white blood cell clones, a process that takes 7-10 weeks. In contrast, the proteomics-based approach takes no more than 3 weeks.

The study also has implications for basic research, such as probing how the antibody response develops, says Polakiewicz. "This is a new technology to develop monoclonal antibodies, so it can be applied for making antibodies for any commercial application, such as research tools, diagnostic assays and therapeutics."

Monya Baker

\section{RESEARCH PAPERS}

Cheung, W.C. et al. A proteomics approach for the identification and cloning of monoclonal antibodies from serum. Nat. Biotechnol. advance online publication (25 March 2012). 